\title{
Etude de la stabilité du lait à l'alcool Solubilité du phosphate et du calcium du lait en présence d'alcool
}

\author{
Alice PIERRE
}

\section{RESUME}

La solubilité des constituants minéraux du lait est diminuée par l'addition d'alcool. La quantité de calcium précipité dépend à la fois de la concentration finale en éthanol et de la teneur en calcium soluble initiale du lait, donc de son $\mathrm{pH}$. La quantité de phosphore précipité est également proportionnelle à la concentration en éthanol, mais peu dépendante de la teneur en phosphore soluble initiale du lait. La précipitation obtenue à partir de laits et de leurs ultrafiltrats a été comparée.

La possibilité que cette précipitation minérale soit le mécanisme de déstabilisation de la micelle en milieu alcoolique est discutée. Mots clés : Alcool - Lait - Phosphore - Calcium.

\section{SUMMARY}

MilK COAGUlation By ALCOHOL

STUDIES ON THE SOLUBILITY OF THE MILK CALCIUM AND PHOSPHATE IN ALCOHOLIC SOLUTIONS

Addition of ethanol decreases solubility of mineral components of milk aqueous phase. Amount of precipitate calcium is evidently related to the ethanol concentration but also to the initial soluble calcium content i.e. $p H$ of the milk. The same phenomena is not observed with phosphorus precipitation which seems only dependant of ethanol concentration. Precipitation of

I.N.R.A., Laboratoire de recherches de technologie laitière, 65, rue de St-Brieuc F 35042 Rennes cedex. 
calcium and of phosphorus upon ethanol addition also behaves differently in milk and its ultrafiltrate. More phosphorus is precipitated when ethanol is added to milk than to ultrafiltrate. Regarding to calcium, the difference is only $1 \mathrm{mM}$.

From the obtained results, the possible relationship between this mineral precipitation which modifies casein micelles - aqueous phase equilibria is discussed notably regarding to the casein micelle destabilization in alcoholic solutions.

Key words :Alcohol - Milk - Phosphorus - Calcium.

\section{INTRODUCTION}

Le test à l'alcool est utilisé traditionnellement pour trier les laits de fromagerie, c'est-à-dire pour déterminer leur aptitude à la coagulation par la présure. Il permet également de sélectionner les laits destinés à la stérilisation, donc de prévoir leur comportement à haute température. La stabilité des micelles de caséine du lait semble être sous la dépendance de mécanismes similaires sous l'action de ces trois facteurs : hydrolyse par la présure, traitements thermiques et addition d'alcool.

La relation entre la composition du lait et la stabilité à l'éthanol a été étudiée par Davies et White (1958) pour un ensemble de laits individuels. Ils ont mis en évidence en particulier une corrélation négative entre teneur en calcium ionisé et stabilité à l'éthanol. Par la suite, Horne et Parker (1981 a, b) ont montré l'importance d'une modification du $\mathrm{pH}$ du lait et de la composition de la phase soluble. Ils ont étudié en particulier les conséquences d'une addition de phosphate ou de calcium. Ils ont également mis en évidence le rôle de la charge de la micelle de caséine (Horne et Parker, 1982).

L'objet de notre étude était de déterminer les modifications de la solubilité du phosphate et du calcium du lait après addition d'alcool, puis d'établir une relation entre cette solubilité et la stabilité des micelles de caséine.

\section{MATERIEL ET METHODES}

Les laits utilisés étaient des laits crus de petit mélange provenant des fermes expérimentales du Ministère de l'Agriculture. Ils étaient écrémés à l'aide d'une écrémeuse centrifuge puis additionnés d'azide de sodium $(0,02 \%)$ comme agent bactériostatique. Dans certains cas, le $\mathrm{pH}$ était modifié par addition de soude $(1 \mathrm{~N})$ ou d'acide chlorhydrique $(3 \mathrm{~N})$. Les ultrafiltrats étaient séparés 
des laits à $20^{\circ} \mathrm{C}$ par ultrafiltration sur un module Romicon équipé de membranes PM 50 de $1,5 \mathrm{~m}^{2}$ de surface membranaire.

Le test de l'alcool était réalisé en mélangeant un volume de l'échantillon, lait ou ultrafiltrat, avec un, deux ou trois volumes d'une solution hydro-alcoolique. Lorsqu'aucune précision n'est donnée, le test était réalisé avec un volume d'échantillon mélangé à deux volumes de solution hydro-alcoolique. Les teneurs en alcool sont exprimées en volume d'alcool pur dans le mélange final. La présence éventuelle d'une coagulation était observée pendant les 5 min qui suivaient le mélange. Lorsqu'une séparation de la phase soluble était souhaitée, un temps de repos de $2 \mathrm{~h}$ à température ambiante était ménagé, puis la séparation était effectuée soit par simple centrifugation (1000 g-30 min) dans le cas des ultrafiltrats, soit par ultrafiltration sur membranes coniques Amicon CF 25, dans le cas des laits (centrifugation 1000 g-1 h).

Le dosage du calcium et du magnésium était réalisé par absorption atomique selon le protocole de Brulé et al. (1974). Le dosage du phosphore était fait selon la méthode de la F.I.L. (1971). Le citrate était déterminé selon la méthode de Pierre et Brulé (1983). Les résultats sont donnés en milligrammes ou en millimoles par litre de solution.

\section{RESULTATS}

\section{A. Stabilité à l'éthanol et calcium soluble du lait}

Dans un lait dont le $\mathrm{pH}$ est modifié par addition de soude ou d'acide, il existe une relation entre la stabilité à l'éthanol et la teneur en calcium soluble. La figure 1 montre que lorsque le calcium soluble augmente de 6,25 à $12,50 \mathrm{mM}$ par $\mathrm{kg}$, sous l'influence d'un changement du $\mathrm{pH}$ de 7,45 à 6,10 , la stabilité à l'éthanol diminue et le pourcentage d'éthanol provoquant la coagulation passe de $66 \%$ à $25 \%$. Davies et White (1958) avaient montré une relation dans le même sens entre stabilité et teneur native en calcium ionisé d'un ensemble de laits.

\section{B. Minéraux solubles résiduels du lait dans les mélanges lait-éthanol}

Trois échantillons d'un lait dont le $\mathrm{pH}$ a été ajusté à 7,45, 6,70 et 6,10 , et les ultrafiltrats séparés des précédents à ces trois $\mathrm{pH}$ ont été mis en présence de mélanges hydroalcooliques à différentes teneurs en alcool. Dans le cas des ultrafiltrats, l'addition d'alcool entraîne l'apparition d'un trouble se transformant progressivement en un pré- 


\section{fig. 1}

Ethanol (p.100)

provoquant la cocgulation

Influence d'une variation de la teneur en calcium soluble du lait (sous l'influence d'un changement de $\mathrm{pH}$ ) sur sa stabilité à l'éthanol.

Les deux points représentés pour chaque échantillon indiquent, pour l'un, la concentration en éthanol la plus élevée sans coagulation, pour l'autre, la concentration la plus faible provoquant la coagulation.

Influence of soluble calcium content of milk (influenced by a pH change) on its 20 ethanol stability.

The two points represented for each sample indicate, one the maximal ethanol concentration without coagulation, the other the lower concentration inducing coagulation.

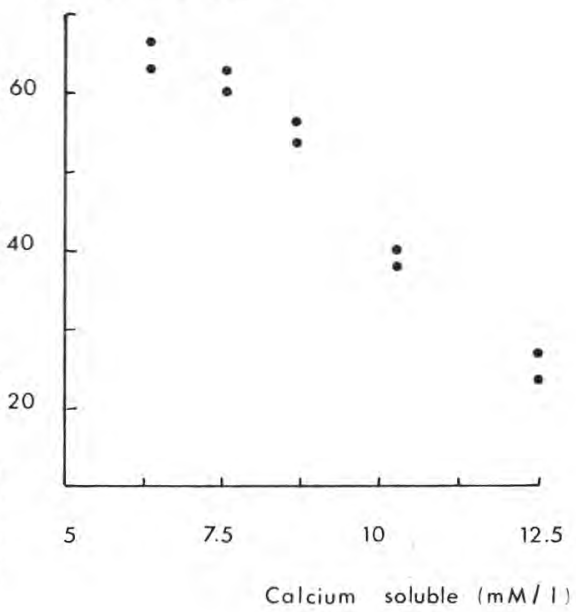

cipité qui sédimente lors de la centrifugation. Dans tous les cas, le $\mathrm{pH}$ des mélanges, échantillon + solution alcoolique, est égal à 7,0. Le pourcentage d'éthanol provoquant la coagulation des laits est de $56 \%$ pour le lait à $\mathrm{pH} 6,70$, de $25 \%$ à $\mathrm{pH} 6,10$; le lait à $\mathrm{pH} 7,45$ ne coagule pas dans un mélange à $67 \%$ d'éthanol.

\section{Calcium}

La teneur en calcium soluble résiduel des mélanges est présentée sur la figure 2. La valeur reportée pour $0 \%$ d'éthanol représente le tiers de la teneur en calcium soluble initiale des produits, en raison de la dilution.

Les laits et les ultrafiltrats sont d'autant plus riches en calcium soluble que leur $\mathrm{pH}$ initial est faible. On observe une décroissance exponentielle des teneurs en calcium soluble résiduel en fonction de la concentration en éthanol. Les mélanges alcooliques de lait ou d'ultrafiltrat au même $\mathrm{pH}$ ont des teneurs en calcium soluble résiduel peu différentes, les laits ont cependant toujours des teneurs légèrement inférieures. On remarque que pour la concentration en éthanol de $10 \%$, il n'y a pas réduction de la teneur en calcium soluble des ultrafiltrats. A une concentration en alcool donnée, la quantité de calcium qui précipite est proportionnelle à la teneur en calcium soluble initiale (fig. 3 ).

\section{Phosphore}

Sur les mêmes échantillons, la teneur en phosphore soluble résiduel a été déterminée (fig. 4). Elle diminue linéairement lorsque la concentration en alcool augmente. L'influence du $\mathrm{pH}$ se traduit 


\section{fig. 2}

Teneur en calcium soluble résiduel des mélanges alcooliques de laits (signes ouverts) ou d'ultrafiltrat (signes fermés) à trois $\mathrm{pH}$ différents : $\mathrm{pH}$ 6,10

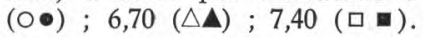

Residual soluble calcium content of alcohol-milk blends (open signs) or alcohol-ultrafiltrate blends (closed signs) at different $p H: 6,10(\bigcirc \bullet) ; 6,70(\triangle \mathbf{\Delta})$; 7,40 ( ).

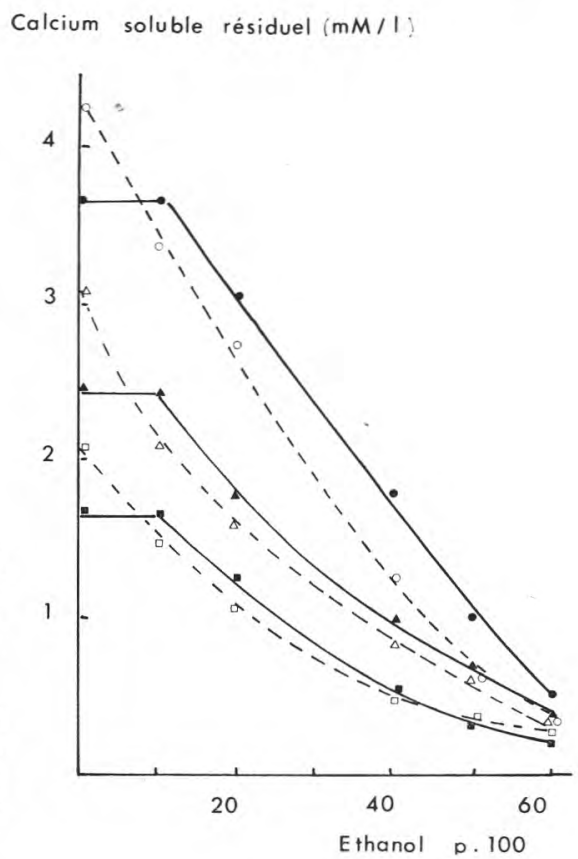

fig. 3

Relation entre la teneur en calcium soluble initiale et la quantité de calcium précipité à partir de laits (signes ouverts) ou d'ultrafiltrats (signes fermés) dans des mélanges alcooliques à $20 \%$ (口) $) ; 40 \%(\triangle \mathbf{\Delta}) ; 60 \%(\mathrm{\bullet})$.

Amount of calcium precipitated from milks (open signs) or ultrafiltrates (closed signs) in alcoholic blends $20 \%$ (口 (⿴) ; $40 \%$ $(\triangle \mathbf{\Delta}) ; 60 \%(\mathrm{O})$ in relation with initial soluble calcium content.

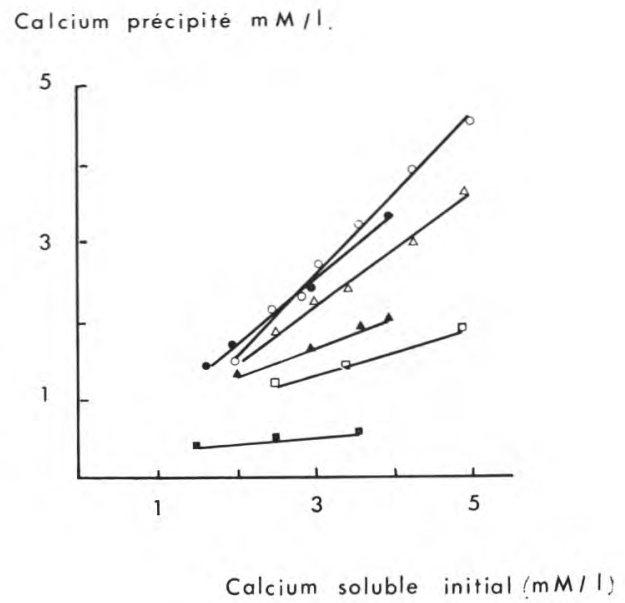

par une différence dans la teneur initiale en phosphore du surnageant, mais a peu d'incidence sur l'allure de la décroissance en fonction de la concentration en alcool. La solubilité du phosphore diminue beaucoup plus vite dans les laits que dans les ultrafiltrats, environ d'un facteur deux, lorsque la teneur en alcool augmente. 
fig. 4

Teneur en phosphore soluble résiduel des mélanges alcooliques de lait (signes ouverts) ou d'ultrafiltrat (signes fermés) à trois $\mathrm{pH}$ différents : $\mathrm{pH} 6,10(\mathrm{\bullet}) ; 6,70(\triangle \mathbf{\Delta})$; 7,40 (무).

Residual soluble phosphorus content of alcohol-milk blends (open signs) or alcohol-ultrafiltrate blends (closed signs) at different $p H: 6,10$

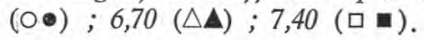

Phosphore soluble résiduel ' $\mathrm{m} M / 1$.

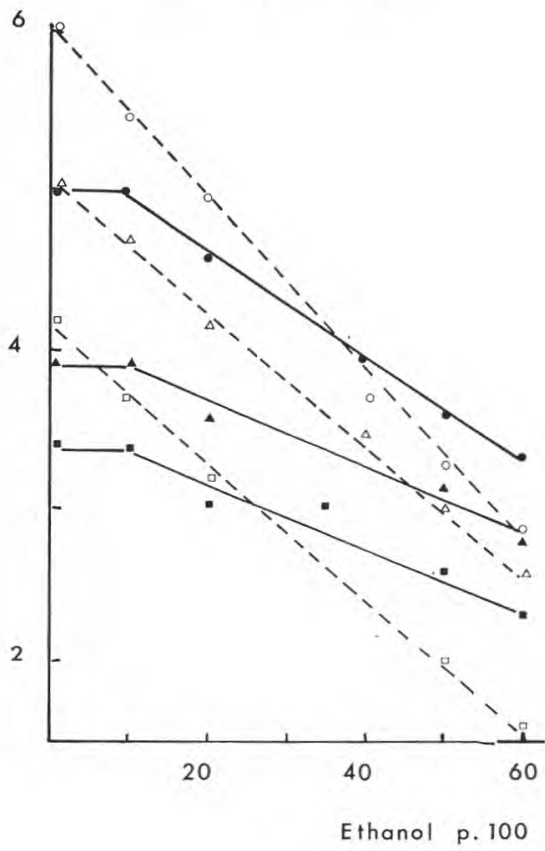

\section{Magnésium}

Le dosage du magnésium dans les surnageants de mélanges alcooliques a permis de mettre en évidence une diminution des teneurs en magnésium. Elle est moins importante dans les ultrafiltrats que dans les laits et elle reste proportionnellement plus faible que celle du calcium. Par exemple, dans un mélange contenant $60 \%$ d'éthanol, la teneur en magnésium diminue de $20 \%$ dans les ultrafiltrats et de $40 \%$ dans les laits, alors que dans les deux cas, celle du calcium diminue de $85 \%$.

\section{Citrate}

Une diminution de la teneur en citrate soluble a été observée dans les laits et les ultrafiltrats. La quantité précipitée est plus importante dans les produits à bas $\mathrm{pH}$ et pour les teneurs en éthanol élevées. Elle est variable selon les laits. Par exemple à $\mathrm{pH} 6,10$ et pour $60 \%$ d'éthanol la quantité de citrate précipité à partir d'un mélange alcoolique est de 0,8 à $1,3 \mathrm{mM}$.

\section{Composition du sel précipité}

Nous avons vu que dans le cas des solutions alcooliques d'ultrafiltrat, la diminution de la teneur en minéraux solubles est accom- 
Calcium précipité $\{\mathrm{mM} / \mathrm{I}$ !

fig. 5

Calcium précipité à partir des mélanges alcooliques de laits (signes ouverts) ou d'ultrafiltrats (signes fermés) à trois $\mathrm{pH}$ différents : $\mathrm{pH}$ 6,10 (०॰) ; $6,70(\triangle \mathbf{\Delta}) ; 7,40(\square \mathbf{\square})$.

Calcium precipitaded from alcoholic blends of milks (open signs) or ultrafiltrates (closed signs) at different $\mathrm{pH}$ : $6,10(\mathrm{O}) ; 6,70(\triangle \mathbf{\Delta}) ; 7,40$ ( $\mathbf{⿴ 囗 十 )}$.
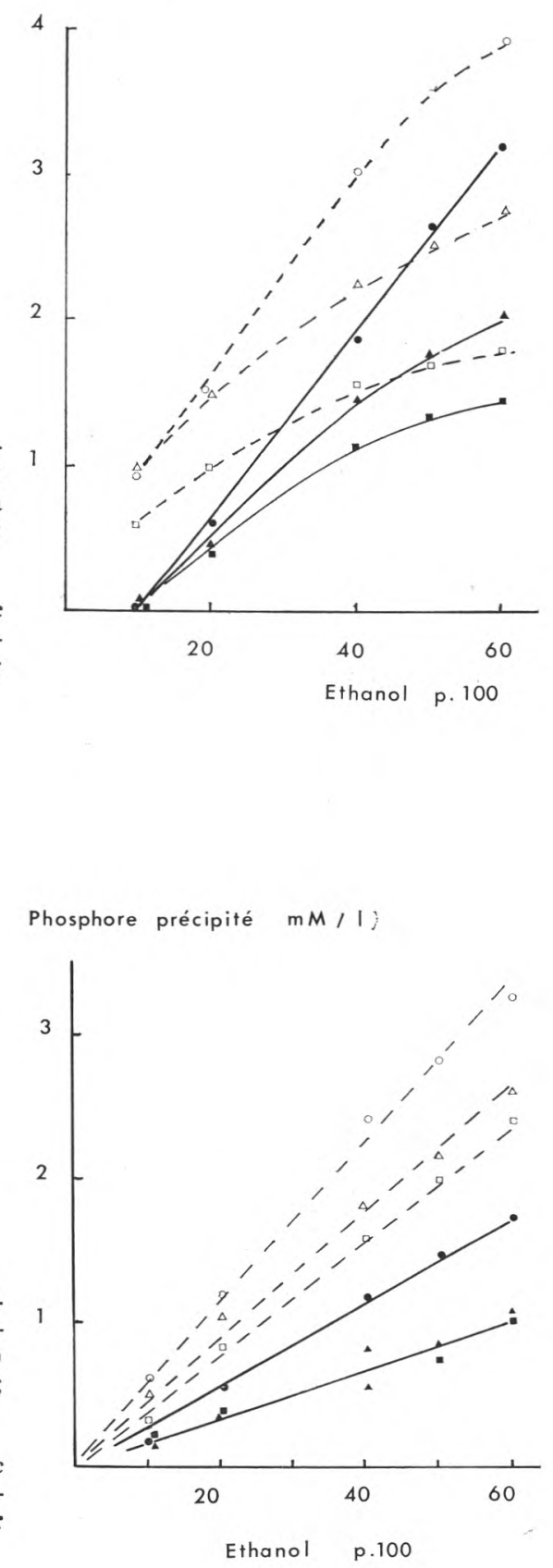

Phosphore précipité à partir des mélanges alcooliques de laits (signes ouverts) ou d'ultrafiltrats (signes fermés) à trois $\mathrm{pH}$ différents : $\mathrm{pH}$ 6,10 $(\mathrm{O} \bullet)$; $6,70(\triangle \Delta) ; 7,40$ (口 घ).

Phosphorus precipitaded from alcoholic blends of milks (open signs) or ultrafiltrates (closed signs) at different $\mathrm{pH}$ : $6,10(\mathrm{O}) ; 6,70(\triangle \mathbf{\Delta}) ; 7,40(\square \mathbf{a})$.

Ethanol p.100 
fig. 7

Rapport molaire du calcium et du phosphore dans les précipités obtenus à partir des mélanges alcooliques d'ultrafiltrats à $\mathrm{pH} 6,10(\bullet) ; 6,70(\mathbf{\Delta})$ et 7,40 (ㅂ) ).

Calcium/phosphorus molar ratio in precipitates obtained from alcoholic blends of ultrafiltrates at $p H \quad 6,10(\bullet)$; 6,70 (1) and 7,40 (.).

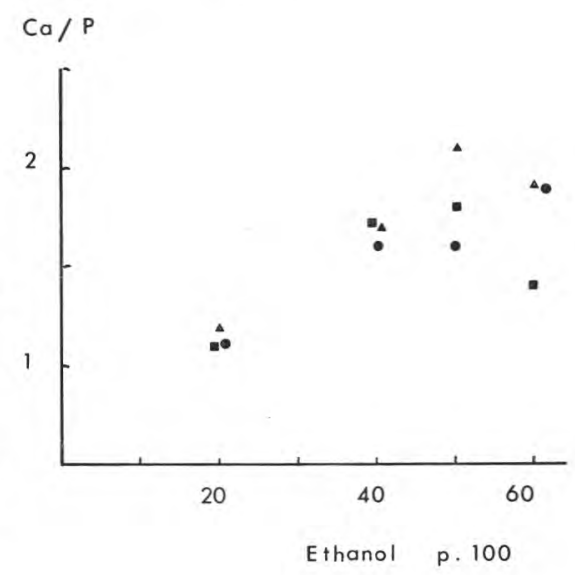

fig. 8

Rapport molaire du calcium et du phosphore dans les précipités obtenus à partir des mélanges alcooliques de laits à $\mathrm{pH}: 6,10(\mathrm{O}) ; 6,70(\triangle)$ et 7,40 (ㅁ).

Calcium/phosphorus molar ratio in precipitates obtained from alcoholic blends of milks at $\mathrm{pH} 6,10(0) ; 6,70$ $(\triangle)$ and $7,40(\square)$.

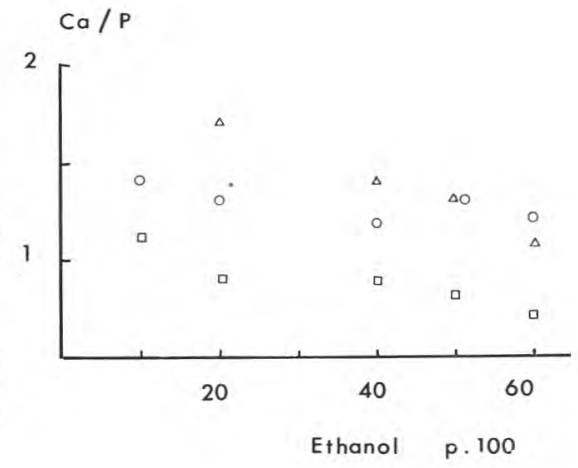

pagnée de l'apparition d'un précipité. Sa composition peut être déduite de la comparaison des teneurs en minéraux solubles de l'ultrafiltrat et des mélanges alcooliques. De même dans les laits, une fraction des minéraux disparaît de la phase soluble, mais il n'est pas possible de mettre en évidence directement la précipitation.

Les quantités de calcium et de phosphore insolubilisés pour chaque teneur en alcool sont reportées sur les figures 5 et 6 .

A un $\mathrm{pH}$ donné, la quantité de calcium insolubilisé à partir du lait est toujours supérieure à celle de l'ultrafiltrat de $1 \mathrm{mM}$ environ; mais l'évolution en fonction de la teneur en éthanol est similaire.

Pour le phosphore au contraire, l'influence de la teneur en éthanol est beaucoup plus forte pour le lait que pour l'ultrafiltrat, 
puisque la quantité de phosphore précipité dans le lait est environ deux fois plus élevée à $\mathrm{pH} 6,10$ et 2,5 fois à $\mathrm{pH} 7,40$ et 6,70 .

Nous avons calculé le rapport molaire $\mathrm{Ca} / \mathrm{P}$ dans chacun des précipités pour toutes les teneurs en éthanol. Pour les ultrafiltrats (fig. 7), ce rapport varie entre 1,1 et 1,9 . Il n'est pas possible de dire d'après nos résultats s'il s'agit d'une augmentation proportionnelle à la teneur en éthanol ou si la valeur du rapport est constante (de 1,6 à 1,9) pour les teneurs en éthanol de 40 à $60 \%$ avec une valeur significativement plus faible, de 1,1 , pour $20 \%$ d'éthanol.

Dans le cas des laits (fig. 8), on observe à la fois une influence de la teneur en alcool et une influence du $\mathrm{pH}$ du lait. Les rapports $\mathrm{Ca} / \mathrm{P}$ sont dans l'ensemble plus faibles que pour les ultrafiltrats ; pour le lait à $\mathrm{pH}$ 6,70, ils varient de 1,4 à 1,2 et à $\mathrm{pH} 7,2$ de 1,1 à 0,7 .

\section{Composition des mélanges à la coagulation. Influence de la nature de l'alcool, de la dilution, du $\mathrm{pH}$}

Nous avons comparé la stabilité du lait $(\mathrm{pH}$ 6,70), vis-à-vis de plusieurs monoalcools, le méthanol, l'éthanol, le n-propanol et d'un polyalcool, le glycérol. Différentes proportions de lait et de mélange alcoolique ont été utilisées, conduisant à une dilution du lait de $0,50,0,33$ ou 0,25 . La teneur en calcium soluble résiduel dans les mélanges provoquant la coagulation a été déterminée et elle est reportée dans le tableau 1 , exprimée soit en concentration, soit par

\section{TABLEAU 1 - TABLE 1}

Caractéristiques du mélange à la coagulation Characteristics of the alcohol-milk blend at coagulation

\begin{tabular}{|c|c|c|c|c|c|c|c|}
\hline $\begin{array}{c}\text { Nature } \\
\text { de } 1^{\prime} \text { alcool }\end{array}$ & $\begin{array}{l}\text { Dilution } \\
\text { du lait } \\
\text { d }\end{array}$ & $\begin{array}{l}\mathrm{Ca} \\
\text { Inftlal } \\
\text { UFxd } \\
(\mathrm{mg} / 1)\end{array}$ & $\begin{array}{l}\text { Alcool } \\
(p .100)\end{array}$ & $\begin{array}{l}\text { Ca } \\
\text { résłduel } \\
(\mathrm{mg} / 1)\end{array}$ & (mg/1) & $\begin{array}{l}\text { précipité } \\
\text { (mg/g caséine) }\end{array}$ & $\begin{array}{c}\text { Constante } \\
\text { diélectrique } \\
\star \star \star\end{array}$ \\
\hline Mé thano1 & $\begin{array}{l}0,25 \\
0,33 \\
0,50\end{array}$ & $\begin{array}{l}104 \\
139 \\
209\end{array}$ & $\begin{array}{c}67 \\
63 \\
50(\mathrm{NC})^{*}\end{array}$ & $\begin{array}{c}16 \\
23 \\
(63)\end{array}$ & $\begin{array}{c}88 \\
116 \\
(145)\end{array}$ & $\begin{array}{c}14,7 \\
14,5 \\
(12,1)\end{array}$ & $\begin{array}{l}47 \\
49 \\
55\end{array}$ \\
\hline Ethanol & $\begin{array}{l}0,25 \\
0,33 \\
0,50\end{array}$ & $\begin{array}{l}104 \\
139 \\
208\end{array}$ & $\begin{array}{l}56 \\
50 \\
42\end{array}$ & $\begin{array}{l}23 \\
35 \\
63\end{array}$ & $\begin{array}{r}81 \\
104 \\
145\end{array}$ & $\begin{array}{l}13,5 \\
13,0 \\
12,1\end{array}$ & $\begin{array}{l}48 \\
51 \\
56\end{array}$ \\
\hline Propano1 & $\begin{array}{l}0,25 \\
0,33 \\
0,50\end{array}$ & $\begin{array}{l}104 \\
139 \\
208\end{array}$ & $\begin{aligned} & 60 \\
& 57 \\
& 50(\mathrm{NC})\end{aligned}$ & $\begin{array}{c}36 \\
41 \\
(84)\end{array}$ & $\begin{array}{r}68 \\
98 \\
(124)\end{array}$ & $\begin{array}{l}11,3 \\
11,7 \\
(10,3)\end{array}$ & $\begin{array}{l}37 \\
39 \\
44\end{array}$ \\
\hline
\end{tabular}

* (NC) : non coagulé.

** : calculées d'après Akerlof (1932). 


\section{TABLEAU 2 - TABLE 2}

Quantité de calcium et de phosphore précipités par l'éthanol ( $\mathrm{mg} / \mathrm{g}$ de caséine) dans les mélanges provoquant la coagulation d'un lait à différents $\mathrm{pH}$

Amount of calcium and phosphorus precipitated by ethanol $(\mathrm{mg} / \mathrm{g}$ casein) in blends inducing coagulation of a milk at different $p H$

\begin{tabular}{l|c|c|c|c}
\hline pH du lait & $\begin{array}{c}\text { \% d'alcool } \\
\text { à la } \\
\text { coagulation }\end{array}$ & $\begin{array}{c}\text { Calcium } \\
\text { précipité }\end{array}$ & $\begin{array}{c}\text { Phosphore } \\
\text { précipité }\end{array}$ & $\begin{array}{c}\text { Ca/P } \\
\text { (rapport } \\
\text { molaire) }\end{array}$ \\
\cline { 2 - 3 } 6,1 & 23 & 8,0 & 5,0 & 1,24 \\
6,4 & 40 & 14,0 & 7,5 & 1,44 \\
6,7 & 57 & 12,0 & 8,5 & 1,09 \\
7 & 63 & 11,9 & 10,0 & 0,92 \\
7,4 & 67 & 9,4 & 11,6 & 0,63 \\
\hline
\end{tabular}

rapport à $1 \mathrm{~g}$ de caséine. Pour un alcool donné, la coagulation se produit pour une même quantité de calcium précipité par gramme de caséine, quelle que soit la dilution par la solution alcoolique. D'un alcool à l'autre, la quantité de calcium précipité à la coagulation est légèrement différente : $11 \mathrm{mg} / \mathrm{g}$ de caséine pour le propanol, 13 pour l'éthanol, 14 pour le méthanol. Sur le tableau figure également la valeur de la constante diélectrique du mélange provoquant la coagulation.

Lorsqu'on fait varier le $\mathrm{pH}$ du lait de 6,10 à 7,40 , la quantité de calcium précipité par l'éthanol dans le mélange provoquant la coagulation évolue et passe par un maximum (tab. 2). La teneur en phosphore précipité est de plus en plus élevée, tandis que le rapport $\mathrm{Ca} / \mathrm{P}$ baisse lorsque le $\mathrm{pH}$ augmente

\section{DISCUSSION}

L'éthanol diminue la solubilité des constituants minéraux et organiques de la phase soluble du lait. Une précipitation apparaît dans les mélanges éthanol-ultrafiltrat. Le précipité d'aspect granulaire contient du calcium et du phosphore, dans un rapport molaire de 1,1 pour les mélanges à $20 \%$ d'éthanol, et dans un rapport 1,7 à 1,9 pour les concentrations supérieures en éthanol. 
La teneur en magnésium de ce précipité est faible. Le mélange hydroalcoolique à $20 \%$ amènerait donc à la formation de phosphate dicalcique, une teneur supérieure en éthanol conduirait à la formation de phosphate tricalcique.

Dans les mélanges éthanol-lait, la précipitation n'a pu être mise en évidence en raison des techniques utilisées. Il est probable que les minéraux insolubilisés se fixent sur les micelles de caséine. La composition du sel précipité évolue avec la teneur en alcool et avec le $\mathrm{pH}$. Quelles que soient les conditions, il y a coprécipitation de calcium, de magnésium et de phosphore. A pH 6,10 et 6,70, le rapport $\mathrm{Ca} / \mathrm{P}$ du précipité est de l'ordre de 1,3, quelle que soit la teneur en alcool, et la contribution du magnésium de 0,1 mole par mole de phosphore. Ces caractéristiques semblent indiquer qu'il se forme un sel de phosphate tricalcique colloïdal. Le phosphate de calcium est le sel qui se forme préférentiellement dans les mélanges alcooliques, en raison de la grande affinité du phosphate pour le calcium. Cependant, en présence d'un excès de calcium (laits à $\mathrm{pH}$ bas), nous avons mis en évidence la précipitation de citrate de calcium. Ce sel est en effet très peu soluble en présence d'éthanol. A pH 7,40 , le rapport $\mathrm{Ca} / \mathrm{P}$ varie de 1,1 à 0,7 . Il ne correspond pas à celui d'un phosphate de calcium pur. Le sel formé pourrait être un sel complexe. La quantité de phosphore soluble qui précipite à partir du lait est deux fois plus grande que celle qui précipite à partir de l'ultrafiltrat dans des conditions comparables. La présence de phosphate minéral colloïdal micellaire et des phosphosérines peut contribuer à augmenter la concentration totale en phosphate du milieu; d'autre part, les protéines apportent des groupements qui agissent sur les équilibres de précipitation du phosphate de calcium, comme les acides glutamiques (Termine et al., 1970).

La stabilité des laits à l'éthanol a été reliée par Davies et White (1958) à l'inverse de leur teneur naturelle en calcium ionique. L'influence d'un changement du $\mathrm{pH}$ du lait a été mise en évidence par Horne et Parker (1980); d'après nos résultats, il semble que l'effet du $\mathrm{pH}$ puisse être expliqué par la variation de teneur en phosphate et en calcium solubles qui en résulte. En effet, le $\mathrm{pH}$ ne semble pas avoir d'influence en tant que facteur physicochimique puisque tous les mélanges hydroalcooliques ont spontanément un $\mathrm{pH}$ égal à 7,00. Par contre, tout changement du $\mathrm{pH}$ du lait fait évoluer l'équilibre de solubilité du phosphate de calcium micellaire. Plus le $\mathrm{pH}$ est élevé, plus la teneur en sels solubles est faible. Or, nous avons mis en évidence que la quantité de minéraux précipités par l'alcool à une concentration donnée est proportionnelle à la teneur initiale en sels solubles. En conséquence, une même proportion d'alcool précipite davantage de minéraux dans les laits acides que dans les laits à $\mathrm{pH}$ élevé. Les sels précipités neutralisent la charge de la micelle. On sait que cette charge est également fonction du $\mathrm{pH}$ (Zittle et al., 1958) : faible à bas $\mathrm{pH}$, augmentant 
progressivement avec le pH jusqu'à 7,2 environ. Pour ces deux raisons, forte teneur en minéraux solubles et faible charge micellaire, la coagulation du lait se produira à des teneurs en alcool d'autant plus faibles que le $\mathrm{pH}$ du lait avant addition d'alcool est plus bas. A pH élevé, la teneur en calcium soluble du lait est si faible que le phosphate ne peut plus précipiter sous forme de phosphate tricalcique, et le lait ne coagule plus quelle que soit la teneur en alcool.

La comparaison des conditions de coagulation d'un lait par des alcools différents a permis d'expliquer la relation déjà observée entre stabilité et constante diélectrique du mélange (Horne et Parker, 1981 c), puisque la solubilité des sels est sous la dépendance de cette propriété des solvants. Cela confirme le rôle de la précipitation minérale dans la stabilité du lait à l'alcool.

\section{REFERENCES BIBLIOGRAPHIQUES}

AKERLOF G., 1932. Dielectric constants of some organic solvent-water mixtures at various temperature. J. Am. Chem. Soc., 54, (11), 4125-4139.

Brulé G., Maubois J.L., Fauquant J., 1974. Etude de la teneur en éléments minéraux des produits obtenus lors de l'ultrafiltration du lait sur membrane. Lait, 54, (539-540), 600-615.

DAviES D.T., White J.C.D., 1958. The relation between the chemical composition of milk and the stability of the caseinate complex. II. Coagulation by ethanol. J. Dairy Res., 25, 256-266.

F.I.L. (Fédération Internationale de Laiterie), 1971. Norme $\mathrm{n}^{\circ} 33$.

HoRne D.S., PARKer T.G., 1980. The pH sensitivity of the ethanol stability of individual cow milks. Neth. Milk Dairy J., 34, 126-130.

Horne D.S., PARKER T.G., 1981 a. Factors affecting the ethanol stability of bovine milk. I. Effect of serum phase components. I Dairy Res., 48, 273-284.

HoRne D.S., PARKeR T.G., 1981 b. Factors affecting the ethanol stability of bovine milk. II. The origin of the pH transition. J. Dairy Res., 48, 285-291.

HORNE D.S., PARKER T.G., 1981 c. Factors affecting the ethanol stability of bovine casein micelles : III. Substitution of ethanol by other organic solvents. Int. J. Biol. Macromol., 3, 399-402.

Horne D.S., PARKER T.G., 1982. Some aspects of ethanol stability of caprine milk. J. Dairy Res., 49, 459-468.

Jenness R., Patton S., 1959. Principles of Dairy Chemistry, New York : Wiley.

Pierre A., Brulé G., 1983. Dosage rapide du citrate dans l'ultrafiltrat de lait par complexation cuivrique. Lait, 63, 66-74.

Termine J.D., Posner A.S., 1970. Calcium phosphate formation in vitro. I. factors affecting initial phase separation. Arch. Biochem. Biophys., 140, 307-317.

Termine J.D., Peckauskas R.A., Posner A.S., 1970. Calcium phosphate, formation in vitro. II. Effects of environment on amorphous-crystalline transformation. Arch. Biochem. Biophys., 140, 318-325.

Zittle C.A., Demmamonica E.S., Rudd R.K., Custel J.H., 1958. Binding of calcium to casein : influence of $\mathrm{pH}$ and calcium and phosphate concentrations. Arch. Biochem. Biophys., 76, 342-353. 